Port Acadie

Revue interdisciplinaire en études acadiennes

An Interdisciplinary Review in Acadian Studies

\title{
Groupe de recherche en études acadiennes
}

\section{Jean-Pierre Pichette}

Numéro 18-19, automne 2010, printemps 2011

URI : https://id.erudit.org/iderudit/1010306ar

DOI : https://doi.org/10.7202/1010306ar

Aller au sommaire du numéro

Éditeur(s)

Université Sainte-Anne

ISSN

1498-7651 (imprimé)

1916-7334 (numérique)

Découvrir la revue

Citer cette note

Pichette, J.-P. (2010). Groupe de recherche en études acadiennes. Port Acadie, (18-19), 171-174. https://doi.org/10.7202/1010306ar d'utilisation que vous pouvez consulter en ligne.

https://apropos.erudit.org/fr/usagers/politique-dutilisation/ 
NOTES DE RECHERCHE

\title{
Groupe de recherche en études acadiennes
}

\author{
Jean-Pierre Pichette \\ Université Sainte-Anne
}

Le 29 août 2005, des professeurs de l'Université Sainte-Anne, principalement issus des départements des sciences humaines, de pédagogie et d'études françaises, ont convenu de former le Groupe de recherche en études acadiennes (GrÉA).

Déjà engagés pour la plupart dans des activités scientifiques, ces chercheurs souhaitaient que le GRÉA soit un lieu de partage de leurs compétences en même temps que d'entraide mutuelle en vue de l'avancement des connaissances en études acadiennes et de leurs recherches en particulier. Ces objectifs découlent de la mission même de l'Université SainteAnne, qui vise au développement de la communauté acadienne et à la promotion de sa culture, par la mise en valeur de l'histoire, de la littérature, de la langue, des traditions et du comportement social des Acadiens et des Acadiennes.

Les membres du GRÉA se proposaient d'accomplir cette mission de diverses façons : par la constitution d'équipes de chercheurs, qui, selon les besoins, sauront s'allier des collègues d'autres universités à l'échelle régionale, nationale ou internationale, par l'organisation de rencontres scientifiques, notamment des conférences, des journées d'études ou des colloques, et par la diffusion de leurs travaux sous les formats appropriés : articles, brochures ou livres autonomes, films, disques ou autres supports.

\section{Réunions scientifiques}

Moins d'un mois après leur formation en unité de recherche, les dixhuit membres fondateurs du GRÉA se sont donné un premier conseil de direction, composé de James Crombie, de Micheline Laliberté, de Maurice Lamothe, de Marc Lavoie, de Jean-Pierre Pichette et du président JeanLouis Robichaud. Et, dès octobre 2005, le GRÉA s'associait à la nouvelle chaire de recherche en oralité des francophonies minoritaires (COFRAM) 
dans l'organisation de ses premières journées d'étude à la Pointe-del'Église sur le thème de la résistance des marges. Quelques mois plus tard, en juin 2006, ils organisaient conjointement un premier colloque : «Le Patrimoine religieux de la Nouvelle-Écosse - Signes et paradoxes en Acadie "; puis un deuxième à l'été 2007 , un colloque international en partenariat avec des universités européennes : "La Résistance des marges »; enfin, à l'automne 2008, c'était des journées internationales d'étude sur «L'Édition des contes de tradition orale».

Toutes ces rencontres scientifiques ont mené à la publication des actes dans Port-Acadie - Revue interdisciplinaire en études acadiennes $(P A)$ qui a pris la relève de la Revue de l'Université de Sainte-Anne:

Le Patrimoine religieux de la Nouvelle-Écosse - Signes et paradoxes en Acadie, actes du colloque organisé les 19 et 20 juin 2006 à l'Université Sainte-Anne, dans Port Acadie, $\mathrm{n}^{\circ}$ 10-11-12, automne 2006 - printemps 2007 - automne 2007, 315 p.

La Résistance des marges - Exploration, transfert et revitalisation des traditions populaires des francophonies d'Europe et d'Amérique, actes du colloque international organisé à l'Université Sainte-Anne du 15 au 18 août 2007, dans Port Acadie, $\mathrm{n}^{\circ} 13-14-15$, printemps 2008 - automne 2008-printemps 2009, $478 \mathrm{p}$.

L'Édition des contes de tradition orale - Pour qui? Comment?, actes des journées internationales d'étude organisées les 23 et 24 octobre 2008 à l'Université Sainte-Anne, dans Port Acadie, $\mathrm{n}^{\circ}$ 16-17, automne 2009-printemps 2010, 273 p.

Il est à noter que, depuis 2008, ces numéros récents de Port Acadie sont dorénavant accessibles en ligne via le portail Érudit : «www.erudit.org/ revues. De même, le GréA s'est doté en 2009 d'un site pour sa revue où on trouve les renseignements nécessaires pour s'y abonner ou connaître ses publications : «www.portacadie.ca〉.

Le GrÉA a également parrainé chacune des conférences de la Cofram où ont été conviés des chercheurs de grande qualité : Ronald Labelle (Université de Moncton), Yves Frenette (Université York), Bernard Émond (Université de Paris), Marlène Belly (Université de Poitiers), André Gervais (Université de Rimouski) et Marcel Bénéteau (Université de Sudbury). 


\section{Travaux des membres}

Les projets de recherche et autres contributions des membres du GRÉA sont annuellement décrits dans le Bulletin du GRÉA. Les trente-cinq chercheurs actuels, qui se recrutent un peu partout en Nouvelle-Écosse (Pointe-de-l'Église, Halifax, Tantallon, Chéticamp), mais aussi au NouveauBrunswick (Moncton, Charlo), à l'île-du-Prince-Édouard (Charlottetown), en Ontario (Toronto, Kingston), au Québec (Trois-Rivières, Montréal) et jusqu'en Louisiane (Lafayette), y ont à ce jour présenté une trentaine de projets, personnels ou en collaboration, ainsi que leurs réalisations (communications, articles, livres, etc.) dans l'un ou l'autre des six numéros du bulletin publiés depuis l'automne 2005 (44 p.). Près de la moitié d'entre eux ont aussi collaboré à une autre revue, également produite depuis 2005 à partir de l'Université Sainte-Anne : Rabaska, revue d'ethnologie de l'Amérique française.

\section{Baccalauréat en études acadiennes}

L'une des premières actions du GRÉA aura été de restaurer le programme d'enseignement en études acadiennes et d'en faire, au moment de sa ratification en avril 2006, le premier baccalauréat à voir le jour dans cette discipline... au monde! Pour le comité d'orientation (Muriel Comeau, Betty Dugas, Micheline Laliberté, Maurice Lamothe, Marc Lavoie et J.-P. Pichette), c'est un grand motif de fierté, puisque ce programme se situe tout à fait dans le prolongement même de la mission de l'Université Sainte-Anne et qu'il met en valeur les assises de l'institution : le Centre acadien, sa bibliothèque et ses importantes archives, le Centre provincial de ressources pédagogiques, la Cofram et son Laboratoire de littérature orale, tout comme le Laboratoire d'archéologie acadienne. En scrutant les divers aspects de l'expérience acadienne, qui est mise en rapport avec la culture des autres régions de la francophonie de l'Amérique et du monde, les cours visent à former une relève polyvalente apte à œuvrer dans des domaines essentiels pour la vitalité de la communauté acadienne : l'enseignement, la recherche, la muséologie, la fonction publique et la communication, sans oublier l'économie. Sept blocs structurent l'enseignement : cours de méthode (1), histoire et archéologie (2), français et littérature (3), ethnologie et folklore (4), linguistique, sociologie et psychologie (5), cours complémentaires (6) et cours de synthèse ou mémoire (7). Ce découpage permet un vaste choix aux étudiants parmi une cinquante cours régulièrement dispensés par le personnel. Après un démarrage lent, le programme paraît plus attrayant et pourrait déboucher dans un avenir qu'on souhaite rapproché sur une première maîtrise en études acadiennes. 
Pour en savoir davantage sur la composition du Groupe de recherche en études acadiennes (membres et conseil de direction) et sur ses activités (travaux, publications), on pourra consulter le Bulletin du GRÉA. 